\title{
Racial and Ethnic Disparities in Obstetrics and Obstetric Anesthesia in the United States
}

\author{
Paloma Toledo $\cdot$ Jorge A. Caballero
}

Published online: 14 September 2013

(C) Springer Science + Business Media New York 2013

\begin{abstract}
Disparities are prevalent within healthcare. Racial and ethnic disparities have been described in the use of neuraxial labor analgesia, in the prevalence of some obstetric conditions, and in obstetric outcomes. The origins of these disparities are likely to be multi-factorial, with patient, provider, and systems-level contributors. One of the objectives of Healthy People 2020 is to achieve health equity, thus eliminating racial and/or ethnic disparities in care. In this review, we give an overview of disparities in obstetric anesthetic and obstetric care.
\end{abstract}

Keywords Disparities - Labor - Neuraxial analgesia · Obstetric outcomes · Patient-provider communication . Race/ethnicity

\section{Introduction}

Racial and ethnic disparities are pervasive in healthcare [1]. There is increasing awareness of the presence of disparities, and taking action to address them has become an

P. Toledo $(\square)$

Anesthesiology and Center for Healthcare Studies, Institute for Public Health and Medicine, Northwestern University Feinberg School of Medicine, 251 E Huron St., F 5-704, Chicago, IL 60611, USA

e-mail: p-toledo@md.northwestern.edu

\section{J. A. Caballero}

Department of Anesthesiology, Pain, and Perioperative

Medicine, Stanford University School of Medicine, Stanford, CA 94305-5640, USA

e-mail: jorgec@stanford.edu issue of national importance. For the past three decades, the US Department of Health and Human Services (HHS) has been releasing the Healthy People objectives. The purpose of Healthy People is to provide evidence-based national objectives to improve the health of all people in the US. Since its inception, one objective within Healthy People has been to address racial and ethnic disparities. In Healthy People 2000 the objective was to reduce disparities; in 2010 the objective was to eliminate disparities; the current objective for 2020 is to establish health equity, thus eliminating health disparities [1]. With $\sim 30 \%$ of the population identifying themselves as belonging to a racial or ethnic minority group, nearly a hundred thousand people are potentially affected by healthcare disparities annually [2]. Disparities have been identified in anesthesia and obstetrics. In this article, we will discuss the various definitions of disparities, and give an overview of disparities in obstetric anesthetic and obstetric care.

\section{Definitions of Disparities}

Multiple definitions of disparities exist. The Agency for Healthcare Research and Quality (AHRQ) defines a disparity as a difference or a gap that exist between two groups [3]. More recently, this definition has been updated to reflect that the differences should be statistically significant and larger than $10 \%$, indicating poor quality or access for the non-referent group (i.e. minority group) [1]. The Institute of Medicine, in contrast, defines a disparity as a difference that is not related to need, preferences, or the appropriateness of an intervention [4]. Finally, the World Health Organization defines a disparity as a difference that is not only unnecessary and unavoidable, but also unfair and unjust [5]. These three definitions fundamentally differ in the acceptability of differences between 
groups. Understanding the definition of disparities used by researchers becomes important in interpreting the results of studies, because not all differences are necessarily disparities if using a more liberal definition of disparities.

Whereas much of the literature discussed in this review focuses on disparities by race/ethnicity, it is important to note that there are several different methods for determining and defining race/ethnicity. Whereas prospective studies usually comment on the methodology used to determine race/ethnicity, this is not specified in most retrospective studies; therefore, the accuracy of the race/ethnicity data, and the effect on the measured disparities are unknown.

For example, one commonly used method involves hospital staff (or research staff) assigning the patients' (or research participants') race/ethnicity by appearance, spoken language, or some other method. The accuracy of this technique was evaluated in one retrospective study in which the race/ethnicity, was assigned to 15,102 patients at a Veterans Administration (VA) outpatient center by hospital staff and was then compared with patients' selfreported race/ethnicity [6]. Hospital staff could select from six categories: Hispanic, American Indian, African American, Asian, White, and unknown and/or missing. The self-reported race/ethnicity data used had been collected for another purpose. Overall, the agreement between assigned race/ethnicity and self-reported race/ ethnicity was best for White patients (76\%), and less for other racial/ethnic groups: $68 \%$ agreement for African American, $57 \%$ for Hispanics, $33 \%$ for Asians, and $1 \%$ for American Indians.

Two other commonly used methods for determining and defining race/ethnicity are surname analysis and geocoding. Surname analysis, as implied, uses patient's last name to identify their race/ethnicity (i.e. a patient with the last name Martinez would be assigned Hispanic ethnicity). Geocoding, recommended by the Institute of Medicine [7], uses geographically coded data from the US Census to characterize people living within that zip code. In one study evaluating diabetes and cardiovascular care disparities, the magnitude of disparities by race/ethnicity were compared by use of geocoded data, and using self-reported race/ethnicity data [8]. The authors found that for all six measures evaluated, the disparities differed by less than one percentage point, suggesting that geocoded data may be used as a reasonable substitute for self-identified race/ ethnicity data.

Self-identification is regarded as the method of choice for data collection; however, with the increasing diversity in the US, patients may identify with more than one racial or ethnic group, and the categorical race options given on forms may not truly be representative of patients' true selfidentified race or ethnicity.
Origins of Healthcare Disparities

One framework for understanding the origins of racial/ethnic disparities was suggested by Kilbourne et al. [9]. Disparities were divided into those that originate at the patientlevel, provider-level, or healthcare system-level. Examples of patient-level disparities are those that arise from differences in patient knowledge or awareness of their treatment options, patient beliefs or treatment preferences, and/or cultural norms. In addition, trust in providers may affect whether patients accept physician's treatment recommendations, and this could contribute to measured disparities in outcomes. Trust in providers has been shown to vary by race/ ethnicity, with African American patients having a greater distrust of physicians than White patients [10]. Disparities also arise at the provider level. For example, a provider may counsel the patient, but in a manner that is not suited to the patient's informational needs, and therefore, the patient may not make an informed medical decision. It is also possible that provider bias exists, and that providers make different recommendations on the basis of patient race/ethnicity. Studies evaluating provider bias have had conflicting results. Schulman et al. [11] demonstrated there was a racial and gender bias in primary care providers' treatment of patients with chest pain. Physicians were asked to view two of six standardized vignettes and make recommendations for the hypothetical patient. In each vignette, the patient's age, gender, and race were varied. Using logistic regression, the authors found than women and African Americans were less likely to be referred to cardiac catheterization than men and Whites, respectively. When race-gender interactions were analyzed, African American women had a lower odds than White men $(0.4 ; 95 \%$ confidence interval, CI $0.2-0.7)$ of being referred for cardiac catheterization. In contrast, Tamayo-Sarver et al. [12], also used standardized clinical vignettes for the management of three conditions (migraines, back pain, and ankle fracture), and did not find a racial/ethnic difference in the prescription of opioids for pain management.

Finally, disparities can also arise at the systems-level. One observational study, which used patient level-data on 13 quality indicators for process of care, found that racial/ ethnic disparities existed for eight of the thirteen performance measures [13]. Hospitals were then stratified into quintiles in each measure (i.e. top-performing hospitals were the top quintile and low-performing hospitals were in the bottom quintile). When logistic regression analysis adjusted for clinical characteristics and hospital site, the magnitude of disparities decreased substantially for most measures, suggesting that disparities may be a function of where minority patients seek care. In all likelihood, disparities are multi-factorial, with contributing patient, provider, and systems-level explanations. 
Disparities in Obstetric Anesthesia

Racial and ethnic disparities in the treatment of both acute and chronic pain have been well documented [14-22]. Addressing disparities in the use of labor analgesia is of great importance, because childbirth is the most common reason for hospital admission in the US [23], and labor is associated with severe pain [24]. Labor pain is most effectively treated with neuraxial analgesia [25]. Two large retrospective studies have revealed racial/ethnic disparity in labor analgesia use, with Hispanic and African American women being less likely to use neuraxial analgesia than White women [26, 27]. In 2004, Rust et al. [26] evaluated neuraxial analgesia use among White, African America, and Hispanic women using the 1998 Georgia Medicaid claims database. Twenty-nine-thousand, eight-hundred and thirty-three women met the inclusion criteria, of which $53 \%$ used neuraxial analgesia. Neuraxial analgesia use was highest for White women, with use of $59.6 \%$. In contrast, Hispanic women had the lowest neuraxial analgesia use, with only $35.3 \%$ choosing to utilize neuraxial analgesia. In a multivariable logistic regression model, race/ethnicity remained a significant predictor of neuraxial analgesia use, even after controlling for age, rural/urban status, and anesthesiologist availability (number of anesthesiologists in practice per 100,000 in the population). Glance et al. [27], using similar methodology, evaluated the New York Perinatal Database for racial and ethnic differences in neuraxial analgesia use. In this retrospective study, 81,883 patients who delivered between 1998 and 2003 were included. A fixed effects logistic regression model was built to test the association between race/ethnicity and neuraxial analgesia use. Similar to Rust, Glance found that African American and Hispanic women were less likely to use neuraxial analgesia than White women, even after controlling for socioeconomic status, provider practice, and clinical characteristics of the women. The Joint Commission has mandated that appropriate pain management is a right for all patients, and an indicator of quality medical care, thus making disparities in analgesic treatment unjust [28].

Although racial/ethnic differences in the use of neuraxial labor analgesia have been documented, the origins of the disparity are not completely understood. As previously mentioned, knowledge of analgesic options by minority patients may contribute to this disparity. Ochroch et al. [29] conducted a telephone survey of 1,193 patients scheduled for surgery at the University of Pennsylvania Hospital. Patients were asked questions regarding their knowledge, experience, and attitudes toward epidural analgesia. Most of the patients stated that they would accept an epidural if recommended by an anesthesiologist (64\%). However, univariate analysis found that patients who were African
American, employed less than part-time, or had a less than a high school education were less likely to accept epidural analgesia than their respective counterparts. In a multivariable logistic regression model, the only variable that remained significant for not using epidural analgesia was African American race (OR 0.58, 95 \% CI 0.41-0.81).

Two studies have evaluated knowledge of analgesic options by using differing methods. The first was a survey study in which patients admitted for labor at the University of Texas hospital were asked about their preferences for labor analgesia and the rationale for their choice [30••]. Twenty-eight participants (14\%) expected to use neuraxial labor analgesia on admission to the hospital, and this number increased to $38 \%$ after analgesic counseling. Patients described a variety of reasons for expecting to avoid neuraxial analgesia, which included: concerns about chronic back pain after neuraxial analgesia (54\%), fears of paralysis (76\%), and friends or family advice against the use of epidural analgesia for labor (36\%). A second study used qualitative methodology to investigate analgesic decision making among 506 parturients admitted for labor at Northwestern Memorial Hospital [31••]. Similar to the previous study, many patients who did not expect to use neuraxial labor analgesia were basing their decision on fears or misunderstandings about the procedure that were not supported by the medical literature [31••]. One of the concerns, fear of back pain after neuraxial analgesia, was voiced by $75 \%$ of the women of Hispanic ethnicity.

In addition to patient knowledge and understanding of analgesic options, other factors may contribute to patientlevel disparities in the use of labor neuraxial analgesia. One population-based surveillance study of 220,814 singleton vaginal deliveries evaluated disparities in neuraxial analgesia use over a two-year period in Canada [32••]. Data from a regional perinatal database were linked to census data to obtain maternal income and educational level. Using generalized estimating equations, the authors found that women from the poorest income quintile were the least likely to receive neuraxial analgesia (odds ratio, OR, 0.59, $95 \%$ CI 0.58-0.91) compared with the richest quintile. Neuraxial analgesia use similarly declined with decreasing educational levels (OR. 0.50, $95 \%$ CI 0.48-0.52).

Another possible contributor to racial and ethnic disparities is health literacy. Health literacy is defined by the Institute of Medicine as the extent to which individuals have the capacity to obtain, process, and understand basic health information and services needed to make appropriate health decisions [33]. Minority adults have been shown to have worse health literacy than White adults [34]. Lowliteracy patients are less likely to receive preventative care or be able to understand medical labels and instructions, and have a higher mortality than high-literacy patients [3538]. To date, the association between health literacy, 
knowledge and understanding of analgesic options, and the use of neuraxial analgesia has not been evaluated.

Culture and pain beliefs also may lead to different neuraxial analgesia use. Use of analgesic medications may be viewed as unacceptable in some cultures. Similarly, in some cultures, pain is viewed as part of the life cycle; therefore, it should be embraced and not treated. One large qualitative study $(n=100)$ evaluated the perceptions of culturally diverse women regarding the experience of pain during childbirth [39]. Women from six regions or countries were interviewed: North and Central America, Scandinavia, the Middle East, China, and Tonga. In many of the cultures, women found meaning in the pain experience. For example, several Hispanic women commented that the experience of the pain helped them feel closer to God; although neuraxial analgesia was available in the hospital, many chose not to use because they felt it might lead to, or be indicative of, other birth complications. In contrast, in the Chinese culture, in which use of neuraxial labor analgesia was similarly unlikely, pain had a more cultural meaning. One Chinese mother said, "although it is painful, it is also easy because women have been having babies for thousands of years," and all mothers must tolerate this pain in order to have a baby.

The second level at which disparities in neuraxial analgesia may potentially originate is that of the care providers. As mentioned previously, there may be differences in the extent of counseling or in the content of analgesic counseling by patient race/ethnicity. It is also possible that the quality of communication between patients and providers differs because of patient race/ethnicity. Patient-centered care is defined as care that is respectful of and responsive to individual patient preferences, needs, and values, and that ensures that patient values guide all clinical decisions [40]. One component of patient-centered care is patient-provider communication, and it has been postulated that differences in this communication contribute to racial and ethnic healthcare disparities [41]. Some studies have revealed disparities in the quality of patient-physician communication [42-44]. One study evaluated patient-provider communication between 61 physicians and 458 African American and White patients [43]. Physicians were found to be $23 \%$ more verbally dominant and $33 \%$ less patient-centered in communication with African American patients than with White patients. These racial and ethnic differences in communication are not inconsequential, because more patient-centered communication has been shown to be associated with better patient recall of information, higher satisfaction with care, and better health outcomes [45-47]. Although the decision to use, or not use, labor analgesia is unique to each patient, with personal expectations, social values, and cultural norms affecting the decision-making process, ineffective communication may leave patients unaware of their analgesic options or fearful of side effects, even if they desire pain relief.

Qualitative research has been used to further understand the origins of disparities from the care provider's perspective. In-depth interviews are used to investigate beliefs, attitudes, and practices. Although no such study has yet been conducted to investigate analgesic disparities, relevant work in the medical setting has investigated provider's beliefs about why disparities occur. Using semistructured interviews with 26 physicians and nurses, four themes were identified:

1 minority patients are less likely to have the same access to care as non-minority patients;

2 the possibility of physician bias toward non-minority patients exists;

3 minority patients mistrust the medical system, so compliance in following provider recommendations is poor; and

4 non-minority patients may demand more care than minority patients [48].

This list is by no means exhaustive, and additional explanations probably exist.

One additional explanation for these disparities is that providers underestimate pain in minority patients, or that providers are not knowledgeable about pain-management. Several studies evaluating management of cancer pain have found that providers tend to underestimate pain in minority populations [16, 49]. One study evaluated ethnic differences in the perception of labor pain. In this prospective observational study, 225 Jewish women and 192 Bedouin parturients were asked to report their pain in labor using an 11-point visual analog scale [50]. A Jewish doctor or midwife also evaluated the patient's pain. Although selfreported pain was similar for Jewish and Bedouin women, the medical providers rated the pain of the Bedouin women as lower than that of the Jewish women (6.89 vs. 8.52, $P<0.001)$. Further study of the effect of language and patient-provider racial/ethnic concordance is still needed in obstetric anesthesia.

Finally, it is possible that systems-level differences may contribute to analgesic disparities. These may be at the level of the hospital or at the health care organization level. Examples of systems-based issues could be lack of providers trained to manage analgesic issues, lack of dedicated anesthesiologists to manage the labor and delivery unit, or lack of the necessary materials for analgesic management. No study has specifically evaluated systems issues as they relate to obstetric analgesic disparities, but extrapolation from the medical literature suggests that this may account for some of the disparities seen. Further work is also needed in this area. 


\section{Obstetric Disparities}

The numerous obstetric disparities that have been described in the literature can be categorized as racial/ethnic disparities that occur in disease incidence, access to care or care process measures, or disparities that occur in medical outcomes. We will briefly discuss some of these disparities that have implications for anesthesiologists.

There is an increased incidence gestational diabetes in women of Hispanic ethnicity [51, 52]. This is probably related to the greater incidence of obesity in the Hispanic population compared with non-Hispanic Whites [53]. Diabetes, gestational diabetes, and obesity are all associated with an increased incidence of macrosomia, shoulder dystocia, and cesarean delivery [54-58]. There also seems to be an increased incidence of preeclampsia in minority women [59, 60]. One retrospective cohort study evaluated racial/ethnic differences in pregnancy outcomes for 32,193 singleton births to parturients with gestational diabetes mellitus in California [59]. In that cohort, the prevalence of preeclampsia was higher for Hispanic and African American women than for non-Hispanic White women. Using multivariable logistic regression, the authors found that African American women were at greater risk of having preeclampsia than women of other races (adjusted odds ratio $1.57,95 \%$ CI 1.47-1.95). A case-control study which evaluated late $(>48 \mathrm{~h}$ ) readmission for preeclampsia similarly found that African American race was associated with an increased risk of hospital readmission for late preeclampsia [60]. Other studies have found ethnic heterogeneity in the prevalence of preeclampsia. One population-based cohort study evaluated 902,460 births in New York City over an eight-year period (1995-2003) [61]. Logistic regression modeling which adjusted for maternal age, education, parity, pre-pregnancy weight, smoking, and year of delivery found that Mexican women were at highest risk of preeclampsia (adjusted odds ratio 2.9, $95 \%$ CI 2.7-3.1) and Hispanic women of Caribbean descent were also at higher risk than non-Hispanic White women (adjusted odds ratio 2.0, $95 \%$ CI 2.0-2.1). It has been suggested that the increased incidence of preeclampsia seen in several of these studies may be because of the confounding effect of comorbid obesity [51]. At least one study observed no racial differences in the incidence of preeclampsia in an obese cohort [62]. It is likely that both social and genetic factors contribute to the apparent increased incidence of preeclampsia in Hispanic and African American women [63-65].

Important racial/ethnic differences have also been found in intrapartum and postpartum conditions and complications. Similar to maternal mortality, the incidence of postpartum hemorrhage has been increasing nationally and internationally [66-70]. In a recent randomized controlled trial evaluating three oxytocin regimens for prevention of uterine atony $(n=1,798)$, Hispanic ethnicity was an independent risk factor for uterine atony that required treatment (odds ratio 2.1, $95 \%$ CI 1.3-3.4), as was nonHispanic White race (odds ratio 1.6, $95 \%$ CI 1.0-2.5) [71]. Similar results have been found in epidemiologic evaluations of disparities in the incidence of obstetric hemorrhage. Using data from the Nationwide Inpatient Sample, a nationally representative database, Bryant et al. [72••] evaluated racial differences in postpartum hemorrhage. In their sample of over two million deliveries there was a higher incidence of postpartum hemorrhage among women of Hispanic ethnicity and Asian/Pacific Islander race. African American women were at increased risk of severe hemorrhage, defined as hemorrhage requiring a blood transfusion or hysterectomy.

Race/ethnicity has been implicated in several important peripartum conditions. African American women have been found to be at increased risk of conditions such as peripartum cardiomyopathy and venous thromboembolic disorders [7375]. For women with peripartum cardiomyopathy, being of African American race is associated with poorer recovery two months following diagnosis [75]. Thrombotic pulmonary embolism is one of the leading causes of maternal mortality in the US [76•]. Death from pregnancy-related venous thrombolic events is $64 \%$ higher for African American women than for women of other races (2.64 deaths per 1,000 deliveries vs. 1.61 deaths per 1,000 deliveries, respectively) [77]. Evaluation of all-cause pregnancy related-mortality reveals a similar racial disparity. Berg et al. [76•] evaluated data from the Pregnancy Mortality Surveillance system from 1998-2005 and found the aggregate pregnancy-related mortality was 14.5 per 100,000 live births, which is the highest seen in the past 20 years. The pregnancy-related mortality ratio for White women was 10.2 , versus 13.4 for women of non-White, non-African-American races, and 37.5 for African American women. The etiology of this fourfold difference in maternal mortality for African American women is unknown.

\section{Conclusion}

Disparities in obstetric anesthesia and obstetric care have been extensively documented. Further understanding the origins of these disparities is important to enable design of strategies to reduce racial and ethnic disparities in care. Solutions will probably need to be multi-faceted, with strategies targeted at the patient, provider, and care-system.

\section{Compliance with Ethics Guidelines}

Conflict of Interest Paloma Toledo and Jorge A. Caballero declare that they have no conflict of interest. 
Human and Animal Rights and Informed Consent This article does not contain any studies with human or animal subjects performed by any of the authors.

\section{References}

Papers of particular interest, published recently, have been highlighted as:

- Of importance

•- Of major importance

1. Agency for Healthcare Research and Quality: 2011 National Healthcare Quality and Disparities Reports. 2012. http://www. ahrq.gov/research/findings/nhqrdr/nhqrdr11/qrdr11.html. Accessed July 2013.

2. Hixson L, Helpler BB, Kim MO. 2010 Census briefs: the white population. 2010. http://www.census.gov/prod/cen2010/briefs/ c2010br-05.pdf. Accessed July 2013.

3. Agency for Healthcare Research and Quality. National healthcare disparities report, 2008, Rockville, MD. 2008. http://www.ahrq. gov/research/findings/nhqrdr/nhdr08/nhdr08.pdf. Accessed July 2013.

4. Institute of Medicine. Unequal treatment: confronting racial and ethnic disparities in health care. Washington: National Academy Press; 2003.

5. Commission on Social Determinants of Health. Closing the gap in a generation: health equity through action on the social determinants of health. Final Report of the Commission on Social Determinants of Health. Geneva: World Health Organization; 2008.

6. Boehmer U, Kressin NR, Berlowitz DR, et al. Self-reported vs. administrative race/ethnicity data and study results. Am J Public Health. 2002;92:1471-2.

7. Ulmer C, McFadden B, Nerenz DR, editors. Institute of medicine: race, ethnicity, and language data: standardization for health care quality improvement. Washington, DC: National Academies Press; 2009.

8. Fremont AM, Bierman A, Wickstrom SL, et al. Use of geocoding in managed care settings to identify quality disparities. Health Aff (Millwood). 2005;24:516-26.

9. Kilbourne AM, Switzer G, Hyman K, et al. Advancing health disparities research within the health care system: a conceptual framework. Am J Public Health. 2006;96:2113-21.

10. Boulware LE, Cooper LA, Ratner LE, et al. Race and trust in the health care system. Public Health Rep. 2003;118:358-65.

11. Schulman KA, Berlin JA, Harless W, et al. The effect of race and sex on physicians' recommendations for cardiac catheterization. N Engl J Med. 1999;340:618-26.

12. Tamayo-Sarver JH, Dawson NV, Hinze SW, et al. The effect of race/ethnicity and desirable social characteristics on physicians' decisions to prescribe opioid analgesics. Acad Emerg Med. 2003;10:1239-48.

13. Hasnain-Wynia R, Baker DW, Nerenz D, et al. Disparities in health care are driven by where minority patients seek care: examination of the hospital quality alliance measures. Arch Intern Med. 2007;167:1233-9.

14. Bernabei R, Gambassi G, Lapane K, et al. Management of pain in elderly patients with cancer. SAGE Study Group. Systematic Assessment of Geriatric Drug Use via Epidemiology. JAMA. 1998;279:1877-82.
15. Chen EH, Shofer FS, Dean AJ, et al. Gender disparity in analgesic treatment of emergency department patients with acute abdominal pain. Acad Emerg Med. 2008;15:414-8.

16. Cleeland CS, Gonin R, Baez L, et al. Pain and treatment of pain in minority patients with cancer. The Eastern Cooperative Oncology Group Minority Outpatient Pain Study. Ann Intern Med. 1997;127:813-6.

17. Cleeland CS, Gonin R, Hatfield AK, et al. Pain and its treatment in outpatients with metastatic cancer. N Engl J Med. 1994;330: 592-6.

18. Epps CD, Ware LJ, Packard A. Ethnic wait time differences in analgesic administration in the emergency department. Pain Manag Nurs. 2008;9:26-32.

19. Heins JK, Heins A, Grammas M, et al. Disparities in analgesia and opioid prescribing practices for patients with musculoskeletal pain in the emergency department. J Emerg Nurs. 2006;32: 219-24.

20. Pletcher MJ, Kertesz SG, Kohn MA, Gonzales R. Trends in opioid prescribing by race/ethnicity for patients seeking care in US emergency departments. JAMA. 2008;299:70-8.

21. Richardson LD, Babcock Irvin C, Tamayo-Sarver JH. Racial and ethnic disparities in the clinical practice of emergency medicine. Acad Emerg Med. 2003;10:1184-8.

22. Todd KH, Samaroo N, Hoffman JR. Ethnicity as a risk factor for inadequate emergency department analgesia. JAMA. 1993;269: 1537-9.

23. Agency for Healthcare Research and Quality. Top five most common reasons for hospital admission in 1996. 2010. http:// www.ahrq.gov/data/hcup/charts/5admiss.htm. Accessed 4 Aug 2010.

24. Brownridge $P$. The nature and consequences of childbirth pain. Eur J Obstet Gynecol Reprod Biol. 1995;59(Suppl):S9-15.

25. Anim-Somuah M, Smyth R, Howell C. Epidural versus nonepidural or no analgesia in labour. Cochrane Database Syst Rev. 2005;4:CD000331.

26. Rust G, Nembhard WN, Nichols M, et al. Racial and ethnic disparities in the provision of epidural analgesia to Georgia Medicaid beneficiaries during labor and delivery. Am J Obstet Gynecol. 2004;191:456-62.

27. Glance LG, Wissler R, Glantz C, et al. Racial differences in the use of epidural analgesia for labor. Anesthesiology. 2007;106:19-25.

28. Lanser P, Gesell S. Pain management: the fifth vital sign. Healthc Benchmarks. 2001;8:68-70, 62.

29. Ochroch EA, Troxel AB, Frogel JK, Farrar JT. The influence of race and socioeconomic factors on patient acceptance of perioperative epidural analgesia. Anesth Analg. 2007;105:1787-92.

30. •- Orejuela FJ, Garcia T, Green C, et al. Exploring factors influencing patient request for epidural analgesia on admission to labor and delivery in a predominantly Latino population. J Immigr Minor Health. 2012;14:287-291. This article evaluates the rational for refusal of epidural analgesia in a predominantly Spanish-speaking Hispanic population. Important patient fears and misconceptions about neuraxial analgesia are revealed.

31. •- Toledo P, Sun J, Peralta F, et al. A qualitative analysis of parturients' perspectives on neuraxial labor analgesia. Int J Obstet Anesth. 2013;22:119-123. This article explores patients' rationale for analgesic decision making using qualitative methodology. Targets for patient education and counseling were identified.

32. • Liu N, Wen SW, Manual DG, et al. Social disparity and the use of intrapartum epidural analgesia in a publicly funded health care system. Am J Obstet Gynecol. 2010;202:273 e271-278. This article evaluates disparities in neuraxial analgesia use in Canada, which has universal healthcare coverage. Even in this setting, socioeconomic disparities in neuraxial analgesia use exist.

33. Institute of Medicine. Health literacy: a prescription to end confusion. Washington, DC: National Academies Press; 2004. 
34. National Center for Education Statistics. The health literacy of America's adults. Results from the 2003 National Assessment of Adult Literacy. Washington, DC: National Center for Education Statistics; 2006.

35. Scott TL, Gazmararian JA, Williams MV, Baker DW. Health literacy and preventive health care use among Medicare enrollees in a managed care organization. Med Care. 2002;40:395-404.

36. Davis TC, Wolf MS, Bass PF 3rd, et al. Literacy and misunderstanding prescription drug labels. Ann Intern Med. 2006;145: 887-94.

37. Wolf MS, Davis TC, Tilson HH, et al. Misunderstanding of prescription drug warning labels among patients with low literacy. Am J Health Syst Pharm. 2006;63:1048-55.

38. Bostock S, Steptoe A. Association between low functional health literacy and mortality in older adults: longitudinal cohort study. BMJ. 2012;344:e1602.

39. Callister LC, Khalaf I, Semenic S, et al. The pain of childbirth: perceptions of culturally diverse women. Pain Manag Nurs. 2003; $4: 145-54$.

40. Institute of Medicine (IOM). Committee on Health Care in America. Crossing the quality chasm: a new health system for the 21st century. Washington, DC: National Academy Press; 2001.

41. Smedley BD, Stith AY, Nelson AR. Unequal treatment: confronting racial and ethnic disparities in health care. Washington, DC: National Academy Press; 2002.

42. Cooper-Patrick L, Gallo JJ, Gonzales JJ, et al. Race, gender, and partnership in the patient-physician relationship. JAMA. 1999;282:583-9.

43. Johnson RL, Roter D, Powe NR, Cooper LA. Patient race/ethnicity and quality of patient-physician communication during medical visits. Am J Public Health. 2004;94:2084-90.

44. Cooper LA, Roter DL, Johnson RL, et al. Patient-centered communication, ratings of care, and concordance of patient and physician race. Ann Intern Med. 2003;139:907-15.

45. Stewart MA. Effective physician-patient communication and health outcomes: a review. CMAJ. 1995;152:1423-33.

46. Roter DL, Stewart M, Putnam SM, et al. Communication patterns of primary care physicians. JAMA. 1997;277:350-6.

47. Hall JA, Roter DL, Katz NR. Meta-analysis of correlates of provider behavior in medical encounters. Med Care. 1988;26: 657-75.

48. Clark-Hitt R, Malat J, Burgess D, Friedemann-Sanchez G. Doctors' and nurses' explanations for racial disparities in medical treatment. J Health Care Poor Underserved. 2010;21:386-400.

49. Anderson KO, Mendoza TR, Valero V, et al. Minority cancer patients and their providers: pain management attitudes and practice. Cancer. 2000;88:1929-38.

50. Sheiner EK, Sheiner E, Shoham-Vardi I, et al. Ethnic differences influence care giver's estimates of pain during labour. Pain. 1999;81:299-305.

51. Ramos GA, Caughey AB. The interrelationship between ethnicity and obesity on obstetric outcomes. Am J Obstet Gynecol. 2005;193:1089-93.

52. Bardenheier BH, Elixhauser A, Imperatore G, et al. Variation in prevalence of gestational diabetes mellitus among hospital discharges for obstetric delivery across 23 states in the United States. Diabetes Care. 2013;36:1209-14.

53. Flegal KM, Carroll MD, Ogden CL, Curtin LR. Prevalence and trends in obesity among US adults, 1999-2008. JAMA. 2010;303:235-41.

54. Kominiarek MA, Vanveldhuisen P, Hibbard J, et al. The maternal body mass index: a strong association with delivery route. Am J Obstet Gynecol. 2010;203:264 e261-267.

55. Vricella LK, Louis JM, Mercer BM, Bolden N. Anesthesia complications during scheduled cesarean delivery for morbidly obese women. Am J Obstet Gynecol. 2010;203:276 e271-275.
56. HAPO Study Cooperative Research Group. Hyperglycaemia and Adverse Pregnancy Outcome (HAPO) Study: associations with maternal body mass index. BJOG. 2010;117:575-84.

57. Makgoba M, Savvidou MD, Steer PJ. The effect of maternal characteristics and gestational diabetes on birthweight. BJOG. 2012;119:1091-7.

58. Casey BM, Lucas MJ, McIntire DD, Leveno KJ. Pregnancy outcomes in women with gestational diabetes compared with the general obstetric population. Obstet Gynecol. 1997;90:869-73.

59. Nguyen BT, Cheng YW, Snowden JM, et al. The effect of race/ ethnicity on adverse perinatal outcomes among patients with gestational diabetes mellitus. Am J Obstet Gynecol. 2012;207: 322 e321-326.

60. Larsen WI, Strong JE, Farley JH. Risk factors for late postpartum preeclampsia. J Reprod Med. 2012;57:35-8.

61. Gong J, Savitz DA, Stein CR, Engel SM. Maternal ethnicity and pre-eclampsia in New York City, 1995-2003. Paediatr Perinat Epidemiol. 2012;26:45-52.

62. Marshall NE, Guild C, Cheng YW, et al. Racial disparities in pregnancy outcomes in obese women. J Matern Fetal Neonatal Med. 2013. doi:10.3109/14767058.2013.806478.

63. Brown HL, Chireau MV, Jallah Y, Howard D. The "Hispanic paradox": an investigation of racial disparity in pregnancy outcomes at a tertiary care medical center. Am J Obstet Gynecol. 2007;197:197 e191-197.

64. Jenkins LD, Powers RW, Cooper M, et al. Preeclampsia risk and angiotensinogen polymorphisms M235T and AGT-217 in African American and Caucasian women. Reprod Sci. 2008;15:696-701.

65. Bodnar LM, Catov JM, Roberts JM. Racial/ethnic differences in the monthly variation of preeclampsia incidence. Am J Obstet Gynecol. 2007;196:324 e321-325.

66. Bateman BT, Berman MF, Riley LE, Leffert LR. The epidemiology of postpartum hemorrhage in a large, nationwide sample of deliveries. Anesth Analg. 2010;110:1368-73.

67. Callaghan WM, Kuklina EV, Berg CJ. Trends in postpartum hemorrhage: United States, 1994-2006. Am J Obstet Gynecol. 2010;202:353 e351-356.

68. Ford JB, Roberts CL, Simpson JM, et al. Increased postpartum hemorrhage rates in Australia. Int J Gynaecol Obstet. 2007; 98:237-43.

69. Knight M, Callaghan WM, Berg C, et al. Trends in postpartum hemorrhage in high resource countries: a review and recommendations from the International Postpartum Hemorrhage Collaborative Group. BMC Pregnancy Childbirth. 2009;9:55.

70. Joseph KS, Rouleau J, Kramer MS, et al. Investigation of an increase in postpartum haemorrhage in Canada. BJOG. 2007; 114:751-9.

71. Wetta LA, Szychowski JM, Seals S, et al. Risk factors for uterine atony/postpartum hemorrhage requiring treatment after vaginal delivery. Am J Obstet Gynecol. 2013;209:51 e51-56.

72. • Bryant A, Mhyre JM, Leffert LR, et al. The association of maternal race and ethnicity and the risk of postpartum hemorrhage. Anesth Analg. 2012;115:1127-1136. This excellent article evaluates racial differences in postpartum hemorrhage from uterine atony.

73. Gentry MB, Dias JK, Luis A, et al. African-American women have a higher risk for developing peripartum cardiomyopathy. J Am Coll Cardiol. 2010;55:654-9.

74. Demakis JG, Rahimtoola SH. Peripartum cardiomyopathy. Circulation. 1971;44:964-8.

75. Amos AM, Jaber WA, Russell SD. Improved outcomes in peripartum cardiomyopathy with contemporary. Am Heart J. 2006; 152:509-13.

76. • Berg CJ, Callaghan WM, Syverson C, Henderson Z. Pregnancyrelated mortality in the United States, 1998 to 2005. Obstet Gynecol. 2010;116:1302-1309. Excellent overview of maternal 
mortality in the United States using the Pregnancy Mortality Surveillance System. There continues to be a racial disparity in maternal mortality, with African American women having a fourfold higher mortality rate than White women.
77. James AH, Jamison MG, Brancazio LR, Myers ER. Venous thromboembolism during pregnancy and the postpartum period: incidence, risk factors, and mortality. Am J Obstet Gynecol. 2006;194:1311-5. 